\title{
L-Dopa and the negative symptoms of Parkinsonism
}

\author{
HAROLD L. KLAWANS, JR. ${ }^{1}$ AND MICHAEL ERLICH
}

From the Presbyterian-St. Luke's Hospital, Chicago, Illinois, U.S.A.

It has been proposed that the neurological abnormalities of Parkinsonism can be divided into positive and negative symptoms (Martin, 1967). Positive symptoms are considered to be release phenomena. Pathological changes within one region of the central nervous system result in the release of this region's control over a second region. The positive symptoms are felt to be related to the physiological dysfunction in this second region. This results from the loss of the normal inhibition of this structure. Examples of positive symptoms are tremor and rigidity.

Negative symptoms are thought to be due to disease of a structure resulting in loss of its normal function. Negative symptoms include difficulty with postural fixation and righting reflexes and akinesia. In humans with Parkinsonism, it has been proposed that 'the degree of damage in the pallidum is sufficient to account for all the loss of postural reflexes' (Martin, 1967).

The purpose of this communication is to review the validity and usefulness of the above classification of symptoms in relation to clinical investigations with the drug L-dopa.

\section{PHARMACOLOGY}

L-Dopa (L-3, 4-dihydroxyphenylalanine) is the immediate precursor of dopamine. Dopamine is the predominant catecholamine in the basal ganglia and substantia nigra (Sano, Gamo, Kakimoto, Taniguchi, Jakesada, and Nishinuma, 1959). In most species dopamine acts as predominantly inhibitory neurotransmitter (Hornykiewicz, 1966). It has also been demonstrated that dopamine has a predominantly inhibitory function in the mammalian caudate nucleus (McLennan and York, 1967).

The present theories of the pharmacology of L-dopa presuppose that its actions are secondary to the formation of dopamine within the central nervous system and that the efficacy of this regime is related to the physiological action of the dopamine

${ }^{1}$ Requests for reprints should be addressed to Dr. H. L. Klawans. formed on the dopaminergic receptors (Barbeau, 1962; Klawans, 1968). According to this theory it would be reasonable to expect that the reinstitution of dopamine inhibition would have a striking effect on the positive symptoms. Evidence as to the effectiveness of L-dopa on rigidity and tremor has been presented by Cotzias, Papavasiliou, and Gellene (1969). L-dopa should, however, have little effect on negative symptoms such as postural reflexes if these symptoms are a reflection of severe destructive lesions of the pallidum.

\section{METHODS}

Patients with known Parkinsonism were hospitalized and taken off standard anti-Parkinsonism medication. Each was begun on 1 to $4 \mathrm{~g}$ oral L-dopa daily and increased to the maintenance dose of 4 to $6 \mathrm{~g}$ daily over a two-week period. The rate of increase and total dose varied according to patient tolerance and response.

Motion pictures were taken of all patients before and during L-dopa therapy. Judgements of postural stability were based on the patient's response to a sudden posterior displacement produced by a push on the sternum with the patient standing as erect as possible. Judgements were made by one of us (H.K.) on reviewing the motion pictures randomly without knowledge as to whether the patient in any movie had yet received L-dopa. Responses were graded as follows: $(0)=$ normal; (1) = retropulsion but recovers unaided; $(2)=$ retropulsion but does not recover unaided; (3) = absence of postural response-would fall if not caught by attendant; (4) = very unstable-tends to fall spontaneously or on turning; $(5)=$ unable to stand without assistance.

\section{RESULTS}

The results of this experiment are shown in the Table.

As evaluated by the sign test, the probability of observing 15 patients with improvement (positive difference) and none with worsening (negative difference) is less than 0.001. Similarly, Student's $t$ test is also significant with a level of confidence of over 0.001 .

The overall results of therapy in these patients will be presented in the future. 
TABLE

RESULTS

\begin{tabular}{lcccc}
\hline No. & Patient & Before L-dopa & On L-dopa & Difference \\
\hline 1 & R.A. & 2 & 0 & 2 \\
2 & F.K. & 4 & 1 & 3 \\
3 & C.K. & 2 & 1 & 1 \\
4 & C.O. & 2 & 0 & 2 \\
5 & R.Y. & 2 & 0 & 2 \\
6 & D.S. & 2 & 0 & 2 \\
7 & H.P. & 3 & 1 & 2 \\
8 & R.H. & 3 & 1 & 2 \\
9 & H.T. & 2 & 1 & 1 \\
10 & E.F. & 3 & 2 & 1 \\
11 & M.C. & 2 & 0 & 2 \\
12 & H.B. & 4 & 1 & 1 \\
13 & D.C. & 2 & 1 & 1 \\
14 & M.K. & 3 & 2 & 1 \\
15 & I.J. & 1 & 0 & 1 \\
16 & J.G. & 1 & 1 & 1 \\
& & & & \\
\hline
\end{tabular}

\section{CONCLUSIONS}

If dopamine acts as an inhibitory neurotransmitter, L-dopa should improve symptoms due to loss of dopamine inhibition - that is, the positive symptoms of Parkinsonism. L-Dopa, however, should have little or no effect on loss of function due to disease or destruction of nervous tissue-that is, the negative symptoms of Parkinsonism.

The above data demonstrate striking improvement in a negative symptom of Parkinsonism, brought about by L-dopa therapy. This result casts doubt on the validity of the present concept of positive and negative symptoms in Parkinsonism and on the one relationship of negative symptoms to destructive lesions. It raises the possibility that so-called negative symptoms are also related to a loss of inhibition or at least to a mechanism that can be reversed by increased central dopamine inhibition.
The observations in this recording relate only to postural reflexes. This symptom was studied because it is the negative symptom whose theoretical pathogenesis and direct relationship to destructive lesions has been best studied. It should also be noted that akinesia which has been suggested to be a negative symptom also responds to L-dopa (Cotzias et al., 1969).

\section{SUMMARY}

The effect of L-dopa on postural reflexes was observed in 16 consecutive patients with Parkinsonism. A striking improvement was noted. This is felt to cast doubt on the theory that the loss of postural reflexes in Parkinsonism is a negative symptom.

L-Dopa was purchased from Nutritional Biochemical Corp., Cleveland, Ohio. This study was made possible by grants from the United Parkinson Foundation, Chicago, Illinois, and its Women's Auxiliary.

\section{REFERENCES}

Barbeau, A. (1962). The pathogenesis of Parkinson's Disease: a new hypothesis. Canad. med. Ass. J., 87, 802-807.

Cotzias, G. C., Papavasiliou, P. S., and Gellene, R. (1969). Modification of Parkinsonism. New Engl.J. Med., 280, 337-345.

Hornykiewicz, O. (1966). Dopamine (3-hydroxytyramine) and brain function. Pharmacol, Rev., 18, 925-964.

Klawans, H. L. (1968). The pharmacology of Parkinsonism. Dis. nerv. Syst., 29, 805-816.

McLennan, H., and York, D. H. (1967). The action of dopamine on neurones of the caudate nucleus. J. Physiol. (Lond.), 189, 393-402.

Martin, J. P. (1967). The Basal Ganglia and Posture. Pitman Medical: London.

Sano, I., Gamo, T., Kakimoto, Y., Taniguchi, K., Jakesada, M., and Nishinuma, K. (1959). Distribution of catecholamine compounds in human brain. Biochem. biophys. Acta (Amst.), 32, 586-587. 